\title{
The moderating influence of self-efficacy on interoceptive ability and counterintuitive decision making in officer cadets
}

DOI 10.1515/jms-2016-0005

Received February 18, 2016; accepted November 17, 2016

Abstract: The rapid technical progress in cyber threats and cyber security poses increased cognitive demands on cyber officers. The macrocognitive demand characteristics placed on the cyber officers exceed those in most common military contexts and are new in nature. Research on decision-making competence within the cyber domain is needed to identify strategies and give a better understanding of how these strategies can have consequences depending on task characteristics. Belief in one's capabilities to handle a certain task has been shown to be a key factor for cognitive performance. This study investigated how high self-efficacy negatively moderated intuitive decision-making tendencies on performance when facing a problem that required counterintuitive strategies.

Twenty-seven cyber officer cadets from the Norwegian Defence Cyber Academy participated in an experiment assessing self-efficacy, interoceptive sensitivity, and decision-making. Participants with high situational self-efficacy generally performed better, but this relationship was moderated by interoceptive sensitivity.

The findings suggest potential detrimental effects of intuitive decision-making tendencies in combination with high self-efficacy. Implications for training and feedback structures in cyber defense are discussed.

Keywords: self-efficacy, decision making, interoception, cardiac accuracy, cognitive performance, cyber defense

\footnotetext{
*Corresponding author: Ricardo G. Lugo, Department of Psychology, Lillehammer University College, Lillehammer, Norway, E-mail: ricardo.lugo@hil.no

Stefan Sütterlin, Department of Psychology, Lillehammer University College, Lillehammer, Norway; Department of Neurobiological Medicine, Oslo University Hospital Rikshospitalet Benjamin J. Knox, Øyvind Jøsok, Kirsi Helkala, Norwegian Defence Cyber Academy, Lillehammer, Norway

Natalie Marie Lande, Department of Psychology, Lillehammer University College, Lillehammer, Norway
}

\section{Introduction}

The rapid progress in cyber threats and cyber security has dominated political thinking to an increased extent and has facilitated the development of national cyber defense strategies in recent years. In network-centric warfare, this has opened space for a new type of astute cyber planner and talented computer network defense operator. These individuals and teams now fulfill a crucial role in defending identified and unidentified network vulnerabilities across countless vectors against unknown threats. Still bound to traditional military structures and lines of command, the new requirements for and the roles of cyber defense officers are yet to be defined (Tikk-Ringas et al. 2014). The cognitive demands required for a successful cyber defense include a cognitive skill set with emphasis on cognitive flexibility, situational awareness, sustained attentional control, and motivation (Helkala et al. 2015a, 2015b, 2016).

The relevance of the human factor in cyber defense has gained the attention of the research community (Mancuso et al. 2014a, 2014b; Gutzwiller et al. 2015; Jøsok et al. 2016). Typical situations in action and training are scenarios marked by time pressure, high complexity, simultaneous and causally unrelated or related events in both the cyber domain and the physical domain, as well as incomplete or potentially deceptive information on which quick decisions and actions have to be based. However, often acting is considered to be not optimal, even where time pressure persists. Acting counterintuitively in relation to time and initiating reflective problem solving - even where more intuitive and spontaneous decision tendencies are strong - require awareness of and control over one's decision-making process. To complicate the matter further, tactical and strategic considerations of the cyber officer are typically constrained by the military command line and the divergent situation awareness, increasing 
the demands for interpretation and communication (Røislien 2015; Williams, 2014).

The cognitive demand characteristics placed on the mostly young cyber officers exceed those in most common military contexts and are new in nature. In cognitive science, these situational demand characteristics are typically related to increased use of "intuitive" decision-making. Klein's (1997) recognition-primed decision (RPD) model describes the concept of intuitive decision-making and explains how people make decisions without having to go through an extensive cognitive process. The RPD describes decision making as a top-down process (pattern matching; intuition) and a bottom-up process (mental simulation; rational process). It is the integration of intuitive and analytical styles and can explain decision-making styles (DMSs) accurately (Klein 1997, 2008; Klapproth 2008).

Intuitive decision making is defined as "affectively charged judgments that arise through rapid, non-conscious, and holistic associations" (Dane and Pratt 2007: p. 33), as opposed to decision-making approaches based on insight or rational reflection (Dane and Pratt 2007). Intuitive decisions can be beneficial in situations marked by complex, incomplete, or deceptive information and time pressure (Strack and Deutsch 2004) when elaborated rational processing is not possible due to restrictions of time and/or cognitive capacity and when emotional information carries task-relevant information (e.g., judging a person's trustworthiness in social interaction, making use of previous implicit learning processes). However, intuitive decision-making can also impair task performance and give rise to irrational and counterproductive decisions where emotional information is task irrelevant and captures needed attentional resources. For the sake of improved task performance in settings where emotional information is task irrelevant, intuition needs to be actively overcome (Alter et al. 2007).

Military personnel operating in cyber defense have been described to continuously switch their domain of action between cyber and physical domains, in a "hybrid space", functioning as core elements simultaneously in sociotechnical and cyber-physical systems (Jøsok et al. 2016). Controlling the emotional effects originating in one domain (e.g., physical domain such as their current social environment) and, thus the potentially detrimental intuitive decisions in another domain (e.g., cyber domain during intruder detection and defense), requires control over decision-making processes and awareness of influencing factors (Jøsok et al. 2016). Among these, overcoming potentially harmful influences of inappropriate reliance on intuition due to lack of either reliable available information or information-processing capacity is part of the new training concepts developed by the Norwegian Defense Cyber Academy (NDCA).

One correlate of cognitive performance that can be subject to training and leadership is self-efficacy. Perceived self-efficacy is the belief in one's competence and abilities to overcome a situation or task (Luszczynska et al. 2005). Bandura (1997) argues that perceived selfefficacy should be conceptualized in a situation-specific manner, but it can also explain a broader range of performance and coping outcomes when the context is less specific. Situational self-efficacy (SSE) is an applied construct and has proven to be a predictor for performance in different domains (Stajkovic and Luthans 1998). Persons with high task-specific self-efficacy show better task performance by choosing better decision-making strategies (Hepler and Feltz 2012). Previous research has shown that self-efficacy can enhance cyber-oriented performance where task demands and their relevance are clearly defined (Choi et al. 2013). These findings suggest that motivation gained by the task-related information increase one's belief in the ability to control the situation and thus facilitates performance.

Enhancing performance in cyber defense scenarios requires consideration of the fact that an attacker has anticipated intuitive countermeasures and incorporated these responses into the attack strategy. Thus, high self-efficacy based on strong beliefs in one's ability to handle a perceived threat might lead to an overconfident reliance on intuitive decision-making. Overconfidence can entail detrimental outcomes in situations where successful problem solving depends on overcoming seemingly correct response tendencies caused by intuitively evoked cognitive biases. In previous research, we have shown that higher degrees of intuitive decision-making assessed via individual differences in interoceptive sensitivity (IS; i.e., strengths of "gut feelings") can be related to higher probability of irrational decisions based on cognitive biases (Sütterlin et al. 2013), whereas the ability to mobilize self-regulatory resources of cognitive control predicts success in counterregulating these confounding influences and enhances performance outcomes in economic decision-making scenarios (Kahneman and Frederick 2006; Sütterlin et al. 2011). However, it remains unclear how these effects are influenced by SSE and whether previous results hold true in a highly selective sample of cyber officer cadets who have undergone demanding selection processes prior to enrollment.

In experimental conditions, a person's tendency to rely on intuitive decision-making can be operationalized via assessment of the individual sensitivity toward visceral 
processes. According to the somatic marker hypothesis (Damasio 1994, 1996), visceral cues or so-called somatic markers are consciously or unconsciously integrated into cognitive decision-making (Ohira 2010; Reimann and Bechara 2010; Suzuki et al. 2003). Neuropsychological research has strengthened the somatic marker hypothesis by the mapping of visceral feedbacks (somatic states) in the insular cortex and their projection onto the ventromedial prefrontal cortex as a crucial brain area for cognitionemotion interaction (Bechara et al. 1999). A large body of research has demonstrated that the ability to make correct decisions in complex situations with incomplete information (e.g., Iowa Gambling Task) is superior in individuals with higher IS (Werner et al. 2009), but task performance is impaired where emotions function as distractors and promote irrational decisions (Sütterlin et al. 2013).

While SSE and IS have previously been shown to facilitate cognitive performance and decision-making, respectively, it remains unclear how these two concepts - a situational experience and a psychophysiological ability relate to each other in regard to decision making under pressure. Klein's (2008) theoretical framework shows validity, but it does not incorporate psychophysiological factors into decision-making paradigms that could help differentiate when emotional information may interrupt pure cognitive decisions. Studies incorporating interoception into the Klein paradigm are missing. Klein's (2008) and Damasio's (1994) theoretical frameworks have overlapping intuitive decision-making aspects, but to date, this integration is only found within clinical research.

The current study investigates the role of high self-efficacy and intuitive decision-making in a contraintuitive decision-making task requiring counterintuitive thinking in a complete cohort of cyber defense officer cadets. Situations in which intuitive decisions and a high confidence in one's own capabilities to handle the situation are present might bear hazardous side effects in cyber defense situations that by nature often require the counterregulation of intuitive influences and engagement of higher reflective cognitive processing under time and performance pressure. We hypothesize that high self-efficacy can give rise to overconfidence and inappropriate reliance on one's intuitive response tendencies and therefore cause a potential vulnerability in tasks requiring counterintuitive problem solving.

The degree of self-efficacy may have a facilitative or debilitative effect on interoceptive ability's influence over decision making. Due to the counterintuitive nature of the dependent variable, we hypothesize that relying on gut feeling combined with high self-efficacy will decrease performance.
H1: High self-efficacy will negatively moderate the relationship between interoceptive ability and decision-making style in a cognitive task.

\section{Methods}

\subsection{Sample description}

Participants comprised 27 Cyber Defence Officer cadets (24 males; mean age $[\mathrm{M}]=21.7$ years; standard deviation $[\mathrm{SD}]=0.71$ ) resembling a complete cohort enlisted in the NDCA. Students accepted for the NDCA undergo a rigorous assessment and selection process focusing on physical fitness, general intelligence, and cyber domain-specific abilities, resulting in considerable homogeneity on numerous measures.

\subsection{Assessment}

\subsubsection{Self-efficacy}

Both trait and SSE scores were assessed. Trait self-efficacy was measured with the General Self-Efficacy Scale (Scholz et al. 2002). The scale is composed of ten Likert-scale items with scores ranging from 1 to 4 , with higher scores indicating higher trait self-efficacy. The scale has shown validity in several domains and across cultures (Luszczynska et al. 2005) and has acceptable internal validity $(\alpha=0.75-$ 0.91). SSE was measured using three scales for positivity, arousal, and confidence. Participants were blind to the cognitive task but had to answer three questions pertaining to it while looking at a visual analog scale that was later coded on a scale of 0-10 (none-very). The three questions were as follows: "How positive are you to the task?", "How aroused do you feel about the task?", and "How confident are you right now?" To compute a state self-efficacy score, the three scales were averaged to a total score, where higher scores indicated higher self-efficacy. This scale followed the guidelines established by Bandura (2006) for creating self-efficacy measurements. The scale created was tailored to the unknown task with unipolar formulations of their current beliefs.

\subsubsection{Decision-making style}

The cognitive reflection test (CRT; Frederick 2005) is a three-item decision-making test. The items consist of 
short logical problems entailing questions with two response options: one response option appears intuitively correct and "obvious" to the reader but is logically wrong, whereas the other answer is correct but requires a deeper reflection and an inhibition of the more "obvious" primary response tendency. Results in the CRT have shown to predict performance in judgment and decision making (Campitelli and Labollita 2010) in the context of heuristics and cognitive biases (Toplak et al. 2011), as well as moral judgments (Paxton et al. 2012). The CRT is scored by adding all correct answers (range: $0-3$ ). The CRT was modified for this study by adding a time limit for the response of 5 seconds for each question to increase ecological validity by inducing psychological stress.

\subsubsection{Interoceptive sensitivity}

IS was operationalized as cardioceptive accuracy, and the assessment followed closely the protocols of Schandry (1981) and Schulz et al. (2013). Participants were asked to count their heartbeat silently without any help of measuring pulse or using devices. Task instructions were verbally presented. The task consisted of four blocks of 15, 35, 45, and 25 seconds, with short resting periods of 30 seconds between trials. Simultaneous verbal and visual cues signaled the start and end of each trial. After each trial, participants were asked the number of perceived heartbeats. Cardiac activity was assessed via photoplethysmographic sensors of the ALIVE ${ }^{\circledR}$ system (SomaticVision, Encinitas, CA, USA) attached to three fingers on the nondominant hand. This method has been validated and used in studies in several domains (Mussgay et al. 1999; Pollatos et al. 2007), and the association with brain areas responsible for intuitive decision making was confirmed with neuroimaging studies (Critchley et al. 2004; Zaki et al. 2012).

\subsection{Session procedure}

Participants were brought from their classroom individually and made to enter a sound-attenuated room where the cardioception task was administered. Participants were then brought to another room where the SSE measurement was taken and CRT was administered. Participants were blind to the purpose of the task and had no prior knowledge of the CRT. They were then instructed that a question would be read aloud to them while also being shown to them simultaneously. After the administrator completed reading the question, they had 5 seconds to respond to the question. If a participant did not respond within the allotted time period, it was recorded as an incorrect answer. No feedback of answer's correctness was given after each question as to not influence self-efficacy states. At the completion of the three questions, participants were debriefed to not communicate any aspects to other participants. All participants participated within a few hours of the start of administration, and communication between participants was made impossible during this time. Participants were debriefed in a group session following testing.

\subsection{Ethical considerations}

Participants signed informed consent prior to the study and were debriefed about the study's purpose after completing the data collection. Participants were informed that they could withdraw from participation at any time and without any consequences throughout and after the session. The study was approved by the Norwegian Social Science Data Services (NSD; project number 43901) and conformed to the NDCA's ethical guidelines for experimental studies.

\subsection{Data reduction and statistical analysis}

Statistical analysis was done with SPSS v.22. The SPSS script for the moderation analysis (PROCESS) was adopted from Hayes (2013) and follows the guidelines established by Baron and Kenny (1986). Pearson's bivariate correlations were calculated, and all variables were entered in the calculation. For the regression analysis, the DMS score was entered as the dependent variable, and the cardiac perception and SSE scores were used as independent variables. Significance level was set to 0.05 (one-tailed for the directed hypotheses). To test for moderation effects of cardioceptive accuracy $(\mathrm{CaC})$ on the relationship between self-efficacy and the DMS, a hierarchical multiple regression analysis was conducted. In the first step, both predictor variables (SSE and IS) were included. In step 2, an interaction variable for "SSE $\times$ IS" was created and added to the regression. If the first step of the regression yields a significant result and the resulting change in $R^{2}$ due to the interaction is significant, then a moderation effect of the predictor variables is in place.

\section{Results}

All trait measurements showed good reliability: general self-efficacy scale (Cronbach's á $=0.748$ ), and participants 
Tab. 1: Descriptive statistics.

\begin{tabular}{lccc}
\hline & Mean (SD) & Minimum & Maximum \\
\hline DMS & $0.74(0.86)$ & 0 & 3 \\
GSES & $28.27(3.28)$ & 19 & 34 \\
SSE & $103.19(57.26)$ & 14 & 252 \\
IS & $0.67(0.23)$ & 0.08 & 0.97 \\
\hline
\end{tabular}

Notes: $N=27 . \mathrm{SD}=$ standard deviation; $\mathrm{DMS}=$ decision-making style; GSES = general self-efficacy scale; SSE = situational selfefficacy; IS = interoceptive sensitivity.

Tab. 2: Pearson's product-moment correlations.

\begin{tabular}{lcccc}
\hline & 1. CRT & 2. GSES & 3. SSE & 4. IS \\
\hline 2. & 0.082 & & & \\
3. & $0.482^{*}$ & -0.065 & & \\
4. & -0.155 & -0.107 & 0.171 & \\
& & & &
\end{tabular}

Notes: $N=27$; DMS = decision-making style; GSES = general selfefficacy scale; $\mathrm{SSE}=$ situational self-efficacy; IS = interoceptive sensitivity.

${ }^{*} p<0.01$ (one tailed).

Tab. 3: Hierarchical regression analysis.

\begin{tabular}{lllllll}
\hline Variable & $\mathbf{B}$ & SE of B & $\boldsymbol{\beta}$ & $\mathbf{T}$ & $\boldsymbol{R}^{2}$ & $\mathbf{F}$ \\
\hline Step 1 & & & & & 0.29 & $4.91^{*}$ \\
SSE & 0.450 & 0.150 & 0.524 & $3.001^{*}$ & & \\
IS & -0.210 & 0.150 & -0.245 & -1.403 & & \\
Step 2 & & & & & 0.39 & $4.93^{*}$ \\
SSE × IS & -0.266 & 0.136 & -0.319 & $1.954^{\star *}$ & & \\
\hline
\end{tabular}

Notes: $\ddot{\mathrm{A}} R^{2}=0.10^{\star *}$. $\mathrm{SE}=$ standard error; $\mathrm{SSE}=$ situational self-efficacy; IS = interoceptive sensitivity.

${ }^{\star} p<0.01$ (one tailed); ${ }^{\star \star} p<0.05$ (one tailed). showed good variance on all scales (Tab. 1). The results of the DMS were comparable to normative data (Fredricks 2003) even with the added time limit restriction, suggesting a generally high cognitive performance level in this sample. General self-efficacy and SSE did not correlate, confirming earlier findings (see Tab. 2; Bandura 1997).

The first step of the hierarchical regression analysis (Tab. 3) showed that the predictors accounted for a significant amount of variance in the DMS scores $\left(R^{2}=0.29\right.$; $F(2,26)=4.91 ; p=0.008)$. The moderator variable, interoceptive perception accuracy, decreased scores on the DMS $(=-0.210 ; t(27)=1.403 ; p=0.087)$ but was not significant. An interaction between the independent variable and the moderator (SSE $\times$ IS) was added to the regression model (step 2), which accounted for a significant change of the variance in DMS scores $\left(\Delta R^{2}=0.101, \Delta F(2,26)=3.819\right.$, $p=0.037 ;=-0.266, t(27)=1.954, p=0.037)$.

The interaction (step 2) was significant, and an examination of the interaction plot was conducted. Analysis showed an enhanced effect that SSE prediction on DMS scores was moderated by IS (Fig. 1). High SSE and low IS predicted higher scores. As interoceptive accuracy increased, DMS scores on high and medium levels of self-efficacy decreased, but for participants with low self-efficacy, high interoceptive accuracy helped increase scores on the DMS slightly. The moderation of IS on SSE, and thus on performance on the cognitive reflection task assessing DMS, supports the hypothesis that intuitive decision making assessed via IS can impair counterintuitive problem solving in the presence of high SSE.

In this study, recruitment possibilities were limited to the number of all available cyber officer cadets in this cohort of the NDCA. Given this limitation, a post hoc power analysis was conducted to determine the achieved power

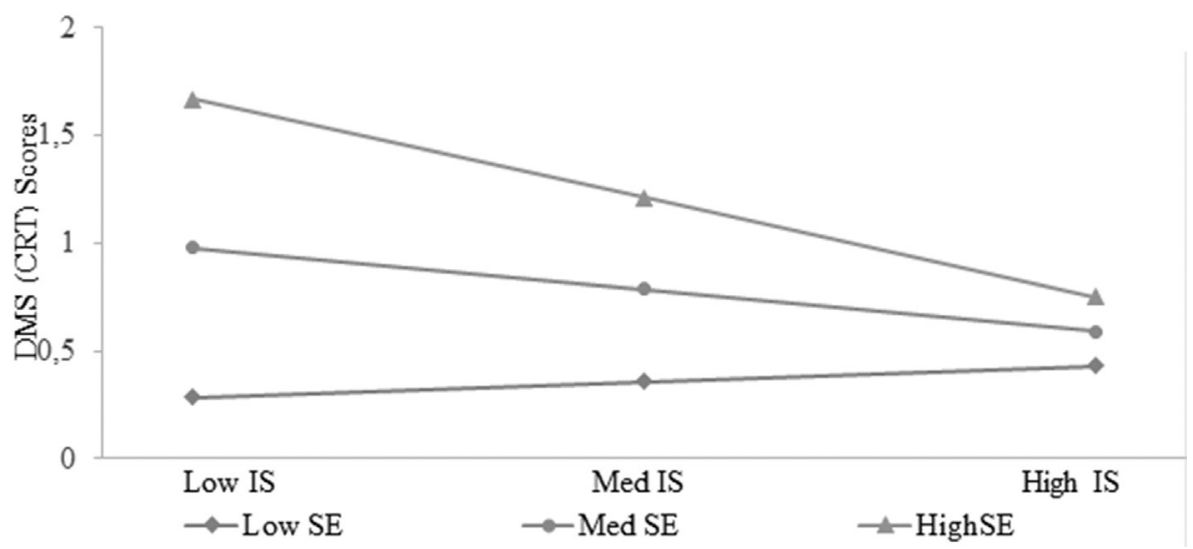

Fig. 1: Interaction effect of self-efficacy (SE) and interoceptive sensitivity (IS) on decision-making style (DMS) (cognitive reflection task or CRT) scores. Low and high IS _ 1 standard deviation from median. 
given a sample size of $N=27$, alpha-level of 0.05 , and the observed effect sizes, resulting in an achieved test power of $80.8 \%$. The minimum required sample size for achieving a test power of the conventionally used $80 \%$ would have been $N=24$ ( $G^{\star}$ Power; Faul et al. 2009). The confidence intervals of the regression were also computed, and the limits at 95\% did not cross zero (lower limit: 0.14 and upper limit: 0.76).

\section{Discussion}

This study investigated the potentially negative influence of high IS (reflecting a person's ability to decide intuitively) in combination with SSE on cognitive performance. The results of the study support the hypothesis that self-efficacy moderated the relationship between interoceptive ability and DMS.

Based on Klein's (2008) conceptualization of intuitive decisions, we applied the Somatic Marker Theory (Damasio 1994) for a more comprehensive understanding of the decision-making process. Previous research has suggested that higher self-efficacy predicts more intuitive decision making (Hepler and Feltz 2012) by increased use of "System 1" processes (Bargh 1994). These decision-making processes are described as unaware, unintentional, resource-economic, and uncontrolled (Bargh 1994; Klein 2008). In earlier studies, it has been demonstrated that a person's sensitivity to interoceptive cues predicts the use of intuitive decision making and can be related to decreased task performance in situations in which an aware, intentional, resource-intensive, and reflective process ("System 2", Bargh 1994) is required for an appropriate decision and overall performance (Sütterlin et al. 2013). In the current study, we extended this line of research in a high-performing sample of cyber officer cadets and investigated the interaction of intuitive decisionmaking tendency as a personality factor (interoception) with subjective SSE in a specific task. The applied cognitive reflection task requires the inhibition of "System 1" cognition for successful task execution, which means that intuitive and seemingly correct obvious answers are wrong, whereas deeper reflection and counterintuitive thinking are required to identify the correct response.

The results of this study suggest an interaction effect of self-efficacy and intuitive decision making. Cyber officer cadets with strong beliefs in their capability to handle a problem and the tendency to often include intuition into their decision making are, under time pressure, more likely to decide for the seemingly easy, but incorrect solution of a given logical but counterintuitive problem. The influence of intuition and related subtle emotional cues happens commonly without awareness. A person's tendency to decide intuitively can be objectively quantified via assessment of sensitivity to interoceptive cues, such as the perception of heartbeats in a resting condition (Schandry 1981). These findings relate to Klein's (1997) research and underline that visceral information is accounted for during decision making in the sense of a bottom-up process.

These findings are in line with previous studies suggesting mediating effects of SSE between personality traits and military competence (Fosse et al. 2015) and correlate with research demonstrating potentially detrimental effects of high self-efficacy on motivation to increase performance in officer cadets (Buch et al. 2015). Our studies connect to and extend these findings by confirming the particular relevance of self-efficacy, adding individual decision-making traits (interoception) to the picture and discussing the practical consequences in a field of rapidly rising importance for the military, namely, cyber defense. Whether higher perceived self-efficacy influences task performance positively depends on the task definition. In situations wherein cognitive heuristics based on intuitive response tendencies and/or emotional information (System 1) are helpful, high self-efficacy and high IS are beneficial. This is typically the case in social interaction, in situations marked by low complexity and with limited response options, or in the context of tactical decision making in conventional infantry. However, in situations in which reflective and controlled counterintuitive decisions are to be made, the combination of high self-efficacy and the high availability of interoceptive information ("gut feelings") impairs decision-making success because these factors increase the probability that an easy but wrong response option is chosen, which is inappropriate for a given complex problem.

These findings are particularly relevant in cyber defense. This becomes evident when taking into account that intuitive and seemingly logical responses, following the detection of an intrusion, can be expected to have been foreseen by an attacker. For example, expected countermeasures are incorporated in the infiltration strategies, which therefore require counterintuitive decision making, the ability to maximize response options, and a high demand on cognitive flexibility.

The results highlight the relevance and need of human factors research in cyber defense. In recent years, human factors have gained increased attention from researchers adding the sociotechnical perspective to the understanding of cyber-physical systems (Gutzwiller et al. 2015; Jøsok et al. 2016). 
Self-efficacy in a task-specific context is the result of previous learning, success, and failure experiences. Individual cognitive processes and abilities, such as decision making and self-efficacy, interact with the experiences of training and feedback by forming response styles in critical situations. Self-efficacy can be represented as both top-down (experience; intuition) and bottom-up (analytical approach) processes and meet the criteria for Klein's (1997) RPD definition. Individual competencies have been neglected in previous research focusing on technical advancement and not including human functioning.

The "Hybrid Space" concept (Jøsok et al. 2016) defines the role of the cyber officer as unique in comparison to other military contexts and provides role descriptions as the discrepancy/asymmetry in situation awareness between cyber officer (situations given in the cyber domain) and commanding officer (situations rooted in the physical domain). The cyber officer is bound to traditional military command structures, challenges traditional roles, and requires increased responsibility and strategical thinking.

Thus, selection, monitoring, training scenarios, feedback, and training of leaders/coaches require a more comprehensive knowledge on factors influencing task performance, in terms of both technical knowledge and skills, as well as cognitive skills, cognitive vulnerabilities, and the psychological determinants of training outcomes. Raising the awareness for this cognitive vulnerability and the ability to analyze given situational demand characteristics should be implemented in training schemes to raise awareness about cognitive failures based on suboptimal choice of decision-making strategies.

There are several limitations to this study. The sample size is of concern. The number of participants (27) is slightly less than the minimum number of 15 participants required per independent variable (30 participants needed for this study), proposed by Park and Dudycha (1974). Furthermore, it is beyond the scope of this study to make any conclusions about the generalizability of these findings for the general public. Compared to samples used in most studies on intuition and decision making, this sample is highly homogeneous in terms of above-average physical fitness and above-average cognitive abilities due to the institutional entry criteria. There might also be systematic differences relative to other samples in terms of variables such as personality traits or motivational factors such as performance orientation. The specific characteristics of this particular sample of military cadets should therefore be kept in mind and replications considered, before conclusions are made regarding other samples. Further research is needed to better understand the determinants and boundaries of cognitive performance and failure in digitized environments embedded in military command structures. Future research should also incorporate rapid task-switching decision-making paradigms for a combined assessment of both "System 1" and "System 2" under high-pressure conditions.

The current study demonstrates how a personality trait, namely, decision making, can interact with results from training and feedback structures, affecting a student's SSE. Inappropriate feedback structures can give rise to either inefficient use of own capabilities (underachievement) - or overconfidence - and the consequential inappropriate reliance on one's intuitive response tendencies and thus cause a potential vulnerability in tasks requiring counterintuitive problem solving. The results highlight the importance of constant monitoring of appropriate task difficulties. Continuous individual adjustments to the task's difficulty levels, or certain aspects of the task, are needed to provide regular outcome assessments. This may increase the individual's metacognitive performance of accurate judgment of one's own performance, which helps to counteract maladaptive levels of overconfidence in one's decision-making strategy.

\section{Acknowledgment}

We thank the cyber officer cadets of the Norwegian Defense Cyber Academy for their dedication and participation in this study.

\section{References}

Alter, A. L., Oppenheimer, D. M., Epley, N., \& Eyre, R. N. (2007). Overcoming intuition: Metacognitive difficulty activates analytic reasoning. Journal of Experimental Psychology: General, 136(4), p. 569.

Bandura, A. (1997). Self-efficacy: The Exercise of Control. Worth Publishers. New York.

Bandura, A. (2006). Guide for constructing self-efficacy scales. In: F. Pajares, \& T. Urdan, (eds.). Self-efficacy Beliefs of Adolescents, Vol. 5. Information Age Publishing, Greenwich, CT, pp. 307-337.

Bargh, J. A. (1994). The four horsemen of automaticity: Awareness, intention, efficiency, and control in social cognition. In: R. Wyer, \& T. Srull (eds.). Handbook of Social Cognition. Lawrence Erlbaum Associates, Inc, Hillsdale, NJ, pp. 1-40.

Baron, R. M., \& Kenny, D. A. (1986). The moderator-mediator variable distinction in social psychological research: Conceptual, strategic, and statistical considerations. Journal of Personality and Social Psychology, 51(6), p. 1173.

Bechara, A., Damasio, H., Damasio, A. R., \& Lee, G. P. (1999). Different contributions of the human amygdala and ventromedial prefrontal cortex to decision-making. The Journal of Neuroscience, 19(13), pp. 5473-5481. 
Buch, R., Säfvenbom, R., \& Boe, O. (2015). The relationships between academic self-efficacy, intrinsic motivation, and perceived competence. Journal of Military Studies, 6(1).

Campitelli, G., \& Labollita, M. (2010). Correlations of cognitive reflection with judgments and choices. Judgment and Decision Making, 5(3), p. 182.

Choi, M., Levy, Y., \& Hovav, A. (2013). The role of user computer self-efficacy, cybersecurity countermeasures awareness, and cybersecurity skills influence on computer misuse. In: Proceedings of the Pre-International Conference of Information Systems (ICIS) SIGSEC - Workshop on Information Security and Privacy (WISP), December 2013, Milan, Italy

Critchley, H. D., Wiens, S., Rotshtein, P., Öhman, A., \& Dolan, R. J. (2004). Neural systems supporting interoceptive awareness. Nature Neuroscience, 7(2), pp. 189-195.

Damasio, A. R. (1994). Descartes' Error: Emotion, Reason, and the Human Brain. Grosset/Putnam Books, New York.

Damasio, A. R. (1996). The somatic marker hypothesis and the possible functions of the prefrontal cortex. Philosophical Transactions: Biological Sciences, 351, pp. 1413-1420.

Dane, E., \& Pratt, M. G. (2007). Exploring intuition and its role in managerial decision making. Academy of Management Review, 32(1), pp. 33-54.

Faul, F., Erdfelder, E., Buchner, A., \& Lang, A.-G. (2009). Statistical power analyses using $\mathrm{G} *$ Power 3.1: Tests for correlation and regression analyses. Behavior Research Methods, 41, pp. 1149-1160.

Fosse, T. H., Buch, R., Säfvenbom, R., \& Martinussen, M. (2015). The impact of personality and self-efficacy on academic and military performance: The mediating role of self-efficacy. Journal of Military Studies, 6(1).

Frederick, S. (2005). Cognitive reflection and decision making. The Journal of Economic Perspectives, 19(4), pp. 25-42.

Gutzwiller, R. S., Fugate, S., Sawyer, B. D., \& Hancock, P. A. (2015). The human factors of cyber network defense. Proceedings of the Human Factors and Ergonomics Society Annual Meeting, 59(1), pp. 322-326.

Hayes, A. F. (2013). Introduction to Mediation, Moderation, and Conditional Process Analysis: A Regression-Based Approach. Guilford Press. New York, New York.

Helkala, K., Knox, B., \& Jøsok, Ø. (2015a). How the application of coping strategies can empower learning. In: Proceedings of Frontiers in Education, El Paso, Texas. pp. 1-8.

Helkala, K., Knox, S., \& Lund, M. (2015b). Effect of motivation and physical fitness on cyber tasks. In: Proceedings of International Symposium on Human Aspects of Information Security \& Assurance, pp. 108-119.

Helkala, K., Knox, B., Jøsok, Ø., Knox, S., \& Lund, M. (2016). Factors to affect improvement in cyber officer performance. Information and Computer Security, 24 (2), pp. 152-163.

Hepler, T. J., \& Feltz, D. L. (2012). Take the first heuristic, self-efficacy, and decision-making in sport. Journal of Experimental Psychology: Applied, 18(2), p. 154.

Јøsok, Ø., Knox, B. J., Helkala, K., Sütterlin, S., Lugo, R., \& Ward, P. (2016). Exploring the hybrid space: Theoretical framework applying cognitive science in military cyberspace operations. In: Schmorrow, D., \& Fidopiastis, C (eds.). Foundations of Augmented Cognition: Neuroergonomics and Operational Neuroscience: Part II. Springer. pp. 178-188.
Kahneman, D., \& Frederick, S. (2006). Frames and brains: Elicitation and control of response tendencies. Trends in Cognitive Sciences, 11(2), pp. 45-46.

Klapproth, F. (2008). Time and decision making in humans. Cognitive, Affective, \& Behavioral Neuroscience, 8(4), pp. 509-524.

Klein, G. (1997). The recognition-primed decision (RPD) model: Looking back, looking forward. Naturalistic Decision Making, pp. 285-292.

Klein, G. (2008). Naturalistic decision making. Human Factors: The Journal of the Human Factors and Ergonomics Society, 50(3), pp. 456-460.

Luszczynska, A., Gutiérrez-Doña, B., \& Schwarzer, R. (2005). General self-efficacy in various domains of human functioning: Evidence from five countries. International Journal of Psychology, 40(2), pp. 80-89.

Mancuso, V. F., Christensen, J. C., Cowley, J., Finomore, V., Gonzalez, C., \& Knott, B. (2014a). Human factors in cyber warfare II emerging perspectives. Proceedings of the Human Factors and Ergonomics Society Annual Meeting, 58(1), pp. 415-418.

Mancuso, V. F., Strang, A. J., Funke, G. J., \& Finomore, V. S. (2014b). Human factors of cyber attacks a framework for human-centered research. Proceedings of the Human Factors and Ergonomics Society Annual Meeting 58(1), pp. 437-441.

Mussgay, L., Klinkenberg, N., \& Rüddel, H. (1999). Heart beat perception in patients with depressive, somatoform, and personality disorders. Journal of Psychophysiology, 13(1), p. 27.

Ohira, H. (2010). The somatic marker revisited: Brain and body in emotional decision making. Emotion Review, 2(3), pp. 245-249.

Paxton, J. M., Ungar, L., \& Greene, J. D. (2012). Reflection and reasoning in moral judgment. Cognitive Science, 36(1), pp. 163-177.

Pollatos, O., Traut-Mattausch, E., Schroeder, H., \& Schandry, R. (2007). Interoceptive awareness mediates the relationship between anxiety and the intensity of unpleasant feelings. Journal of Anxiety Disorders, 21(7), pp. 931-943.

Reimann, M., \& Bechara, A. (2010). The somatic marker framework as a neurological theory of decision-making: Review, conceptual comparisons, and future neuroeconomics research. Journal of Economic Psychology, 31(5), pp. 767-776.

Røislien, H. E. (2015). When the generation gap collides with military structure: The case of the Norwegian cyber officers. Journal of Military and Strategic Studies, 16(3). pp. 23-44.

Schandry, R. (1981). Heart beat perception and emotional experience. Psychophysiology, 18(4), pp. 483-488.

Scholz, U., Doña, B. G., Sud, S., \& Schwarzer, R. (2002). Is general self-efficacy a universal construct? Psychometric findings from 25 countries. European Journal of Psychological Assessment, 18(3), p. 242.

Schulz, A., Lass-Hennemann, J., Sütterlin, S., Schächinger, H., \& Vögele, C. (2013). Cold pressor stress induces opposite effects on cardioceptive accuracy dependent on assessment paradigm. Biological Psychology, 93(1), pp. 167-174.

Stajkovic, A. D., \& Luthans, F. (1998). Self-efficacy and work-related performance: A meta-analysis. Psychological Bulletin, 124(2), p. 240.

Strack, F., \& Deutsch, R. (2004). Reflective and impulsive determinants of social behavior. Personality and Social Psychology Review, 8(3), pp. 220-247. 
Sütterlin, S., Herbert, C., Schmitt, M., Kübler, A., \& Vögele, C. (2011). Frames, decisions, and cardiac-autonomic control. Social Neuroscience, 6(2), pp. 169-177.

Sütterlin, S., Schulz, S. M., Stumpf, T., Pauli, P., \& Vögele, C. (2013). Enhanced cardiac perception is associated with increased susceptibility to framing effects. Cognitive Science, 37(5), pp. 922-935.

Suzuki, A., Hirota, A., Takasawa, N., \& Shigemasu, K. (2003). Application of the somatic marker hypothesis to individual differences in decision making. Biological Psychology, 65(1), pp. 81-88.

Tikk-Ringas, E., Kerttunen, M., \& Spirito, C. (2014). Cyber security as a field of military education and study. Joint Force Quarterly, 74(3), pp. 57-60.

Toplak, M. E., West, R. F., \& Stanovich, K. E. (2011). The Cognitive Reflection Test as a predictor of performance on heuristicsand-biases tasks. Memory \& Cognition, 39(7), pp. 1275-1289.

Werner, N. S., Jung, K., Duschek, S., \& Schandry, R. (2009). Heartbeat detection and the experience of emotions. Cognition and Emotion, 14(3), pp. 417-427.

Williams, B. T. (2014). The joint force commander's guide to cyberspace operations. Joint Force Quarterly, 73, 2nd Quarter, 2014.

Zaki, J., Davis, J. I., \& Ochsner, K. N. (2012). Overlapping activity in anterior insula during interoception and emotional experience. Neuroimage, 62(1), pp. 493-499.

\section{Biographies}

Ricardo G. Lugo is a lecturer in psychology at Lillehammer University College. His research interests involve health and performance psychology, with a focus on aspects of developmental, behavioral, and emotional regulation of performance in several domains.

Stefan Sütterlin is a professor in Psychology at Lillehammer University College and affiliated with Oslo University Hospital, Norway. His research interests include psychophysiology of behavioral control, decision-making, and cognition-emotion interaction.

Kirsi Helkala is an associate professor at the Norwegian Defense Cyber Academy, holding a PhD in Information Security from the University of Oslo, Norway. Her research interests lie in information and cyber security, especially its human aspects.

Benjamin J. Knox is an instructor in International Studies at the Norwegian Defense Cyber Academy currently studying MSc in Development Management, and his research interests lie in improving human performance in digitization.

Lieutenant Commander Øyvind Jøsok is an instructor in Leadership at the Norwegian Defense Cyber Academy. He holds an MSc in leadership and is currently a PhD candidate within the area of cognitive performance psychology. His research interest lies in the area of physical and cognitive development and performance.

Natalie Marie Lande studies Psychology at Lillehammer University College. She has experience in the Norwegian Armed Forces and works as a research assistant and coordinator in a collaborative research project between Lillehammer University College and the Norwegian Defense Cyber Academy. 